\title{
Linear stability analysis of a counter rotating vortex pair of unequal strength
}

\author{
$\begin{array}{lll}\text { J. So } & \text { K. Ryan } & \text { G. J. Sheard } \\ & \end{array}$
}

(Received 15 August 2008; revised 7 October 2008)

\begin{abstract}
An elliptic type instability of a counter rotating vortex pair of unequal strength was numerically investigated with a linear stability analysis method. The peak growth rates of the unstable modes were predicted. The instability characteristics were found to differ from an equal strength vortex pair, of either co-rotating or counter rotating vortices. This investigation serves as a fundamental model to the flow of two unequal strength vortices, which can be generated from the ends of aerodynamic surfaces of an aircraft, such as wing tips and ailerons. These results provide predictions of the vortex arrangements likely to develop three dimensional instabilities, which is known to promote the dissipation of the underlying vortex structure.
\end{abstract}

http://anziamj.austms.org.au/ojs/index.php/ANZIAMJ/article/view/1455 gives this article, (c) Austral. Mathematical Soc. 2008. Published October 29, 2008. ISSN 1446-8735. (Print two pages per sheet of paper.) 


\section{Contents}

1 Introduction

C138

2 Numerical approach

C139

2.1 Numerical model-baseflow computation . . . . . . . . . . C140

2.1.1 Grid resolution independence . . . . . . . . . . . C141

2.1.2 Adaptation of the vortices . . . . . . . . . . C C142

2.2 Linear stability analysis . . . . . . . . . . . . C C143

3 Stability analysis results

C145

4 Conclusion

C148

References

C149

\section{Introduction}

The instability of an equal circulation strength vortex pair (either co-rotating or counter rotating) has been the subject of intense investigation in the past $[6,7,9$, e.g.]; owing to its potential application in the aviation industry [15]. Generally, investigations have identified an elliptic instability to be the mechanism driving the perturbation growth on the vortex pair [4]. The elliptic instability was first identified by Widnall \& Sullivan [16] on a vortex ring and was classified as a short wave instability, as distinct from the Crow (or long wave) instability [2]. An analytical model for the short wave instability was developed by Widnall et al. [17] for a vortex ring. They also argued that an analogous instability mechanism can support the shortwave instability on a vortex pair. The argument was supported by Moore \& Saffman [10] who concluded that providing a finite strain at the vortex core was an essential condition to the growth of a short wave instability on a straight vortex filament. The strain field itself deforms the (initially) circular 
vortex profile into an elliptic one. The underlying strain field was shown to excite the resonance of two Kelvin modes [3] leading to the growth of the elliptic instability.

The linear elliptic instabilities have been shown to lead to dramatic nonlinear instability interaction, which enhance the circulation mixing between two vortices and reduce the vortex pair coherence. Such phenomena was observed by Leweke, Williamson and Meunier [7, 9], and the instability was suggested to be applicable for reducing the long life of aircraft wake vortices.

The instability of an unequal strength vortex pair has had significantly less attention. This article addresses this issue by considering the linear instability growth on a counter rotating, unequal strength (CRUS) vortex pair when the circulation ratio is varied. The numerical approach is described in Section 2 and investigation results presented in Section 3.

\section{Numerical approach}

The numerical approach begins with a two dimensional (2D) baseflow computation (Section 2.1) followed by a linear stability analysis (Section 2.2) of the 2D flows to the growth of three dimensional (3D) perturbations. A 2D baseflow was first resolved toward a quasi-steady state [14] under the two dimensional Navier-Stokes equations and the continuity equation

$$
\begin{aligned}
\frac{\partial \mathbf{u}}{\partial \mathrm{t}}+(\mathbf{u} \cdot \nabla) \mathbf{u} & =-\nabla p+v \nabla^{2} \mathbf{u}, \\
\nabla \cdot \mathbf{u} & =0 .
\end{aligned}
$$

Here, $\mathbf{u}=(u(x, y, t), v(x, y, t))$ is the velocity vector, $p$ is the kinematic static pressure scalar and $v$ is the kinematic viscosity. The resolved 2D baseflow was then analyzed with a linear stability analysis technique, described in Section 2.2. 

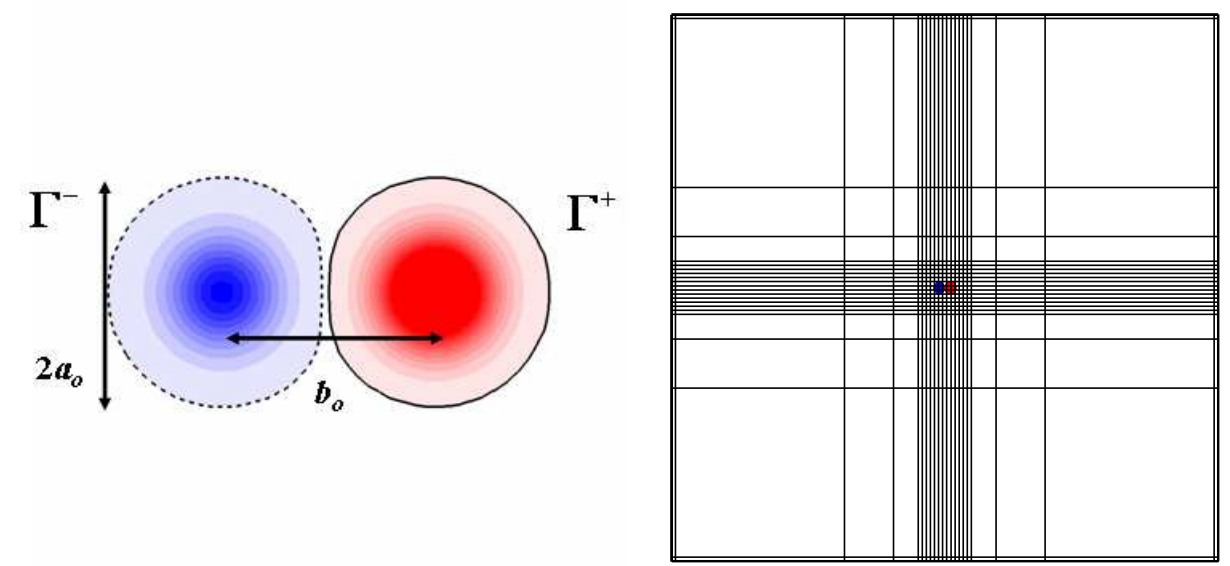

Figure 1: Left: Schematic diagram of a vortex pair with $\Lambda=-0.51$. Right: Current mesh showing the superimposed vortex pair.

A spectral element method was applied to spatially discretize the domain in this investigation. Time integration was handled by a third order accurate backwards multi-step scheme. Sheard et al. [12] and So et al. [14] recently employed this algorithm and provide many details.

\subsection{Numerical model-baseflow computation}

The vortex pair, as in Figure 1 (left), is described with spacing ratio $a_{0} / b_{0}$, where $a_{0}$ and $b_{0}$ are the initial values of the vortex radius and the vortex center separation respectively; and a relative initial circulation strength ratio, $\Lambda=\Gamma_{0}^{-} / \Gamma_{0}^{+}$, where $\Gamma_{0}$ is the vortex initial circulation; and the positive and negative indicate the circulation sign of each vortex. The Reynolds number $\operatorname{Re}=\Gamma / v$ of the flow is defined based on the initial $\Gamma_{0}^{+}$. Table 1 summarizes all flow parameters. Each vortex initially has a Gaussian vortex profile, where 
TABle 1: Parameter space of the baseflow computation

\begin{tabular}{ccccc}
\hline $\operatorname{Re}$ & $a_{0}$ & $a_{0} / b_{0}$ & $\Gamma_{0}^{+}$ & $\Lambda$ \\
20000 & 1 & 0.25 & $3 \pi$ & $\in[-1,-0.1]$ \\
\hline
\end{tabular}

the axial vorticity $\omega_{\text {axial }}$ is defined in polar coordinates as

$$
\omega_{\text {axial }}=\frac{\Gamma_{0}}{\pi \mathrm{a}_{0}^{2}} e^{-\left(\mathrm{r} / \mathrm{a}_{0}\right)^{2}},
$$

where $r$ is the radial coordinate. A Gaussian vortex has the advantage that its profile remains invariant over time despite viscous effects. The vortex size grows in time following $a_{t}=\sqrt{4 v t+a_{0}^{2}}[1]$, where $t$ is time. This vortex profile has been employed in several past investigations [5, 6, e.g.]. Figure 1 (right) shows two superimposed Gaussian vortices in the current domain with a size of $100 a_{0} \times 100 a_{0}$.

\subsubsection{Grid resolution independence}

The spatial accuracy of a spectral element method is dependent on the order $n$ of the Lagrangain polynomials employed on the grid. A grid resolution study was performed to determine sufficient resolution during the baseflow computation with a CRUS vortex pair of $\Lambda=-0.5$ and $a_{0} / b_{0}=0.25$ at $\operatorname{Re}=20000$. Polynomials of $n=10$ were consequentially chosen to apply in all simulations in the investigation, which provided the strain rate and the vorticity measurements at the vortex center with less than $0.05 \%$ deviation from the highest resolution study, $n=12$. A time step of $\Delta t=0.001$ was found to be stable for all simulations. 

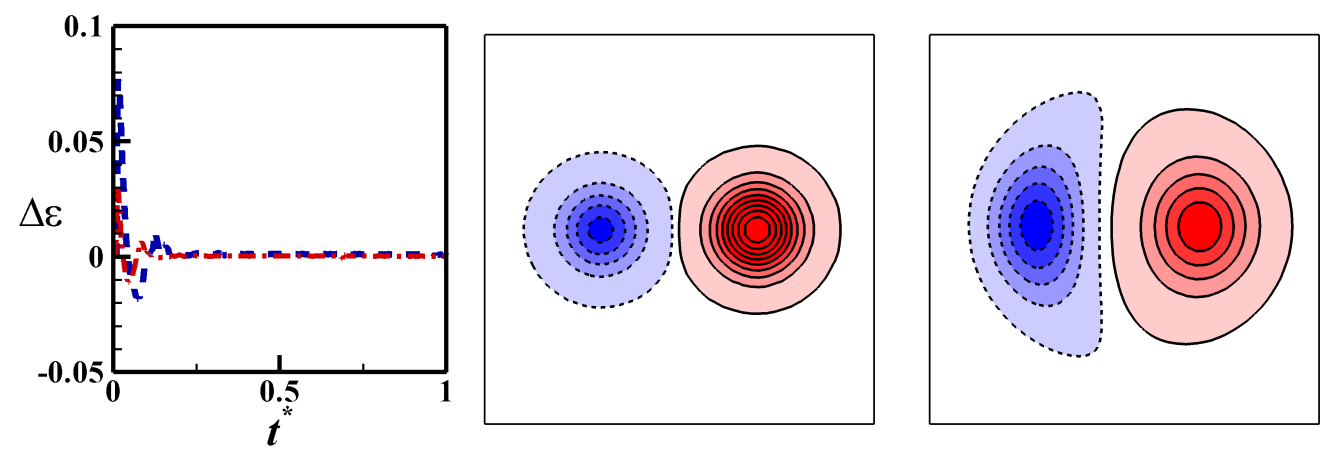

Figure 2: Left: Rate of change of $\varepsilon$ for a $\Lambda=-0.51$ vortex pair over one period of self-rotation. Middle: Initial superimposed vortices. Right: Adapted vortices at $t^{*} \approx 1$. Flooded contours of normalized axial vorticity are plotted. Red and blue contours show positive and negative vorticity respectively.

\subsubsection{Adaptation of the vortices}

Two superimposed Gaussian vortices are not a solution of the Navier-Stokes equations. The vortices have to adapt to the mutually induced strain and such an adaptation process has been shown to be a 2D inviscid process for equal strength vortex pairs [8]. The 2D flow computation of the vortex pair was resolved with (1) and (2) toward a quasi-steady solution, which satisfies the Navier-Stokes equations. So et al. [14] found that a longer adaptation time was required for the weaker vortex of a CRUS vortex pair.

In the current study, the eccentricity of each vortex was monitored during the adaptation. The eccentricity $\varepsilon$ measures how much a vortex has deformed within an external strain field. Here, the eccentricity $\varepsilon=2 S_{i} / \omega_{\text {axial }}$ following the work of Le Dizés \& Verga [8], where $S_{i}$ is the internal strain rate, due to the strain imposed by the profile of the deformed vortex on itself. Figure 2 (left) shows the variation of $\Delta \varepsilon$ over time for the case of $\Lambda=-0.51$; the form of this plot is typical of that found across all values of $\Lambda$ considered. Figure 2 (middle \& right) shows contours of vorticity both 
prior to the adaptation $\left(t^{*}=0\right)$ and after the adaptation $\left(t^{*} \approx 1\right)$, where $\mathrm{t}^{*}=\mathrm{t} / \mathrm{T}=\mathrm{t}\left(\Gamma^{+}+\Gamma^{-}\right) / 4 \pi^{2} \mathrm{~b}^{2}$. The simulation was carried out at $\mathrm{Re}=6600$ to show the viscous growth in the vortex radii with $\approx 30 \%$.

\section{$2.2 \quad$ Linear stability analysis}

The global instability modes and accompanying growth rates were computed by a linear stability analysis method. The perturbations were evolved on a frozen flow field to eliminate the effect of viscous diffusion of the vortex cores. The linearised Navier-Stokes equations $(4,5)$ were integrated forward in time and formed the governing equations for the perturbation evolution. All computations in the stability investigation were carried out at $\operatorname{Re}=$ 20000. Lacaze et al. [5] employed the frozen baseflow approach to investigate the elliptic instability of a counter rotating equal strength (CRES) vortex pair with axial flows.

$$
\begin{aligned}
\frac{\partial \mathbf{u}^{\prime}}{\partial \mathrm{t}}+(\overline{\mathbf{U}} \cdot \nabla) \mathbf{u}^{\prime}+\left(\mathbf{u}^{\prime} \cdot \nabla\right) \overline{\mathbf{U}} & =-\nabla \mathrm{P}^{\prime}+v \nabla^{2} \mathbf{u}^{\prime}, \\
\nabla \cdot \mathbf{u}^{\prime} & =0,
\end{aligned}
$$

where $\mathbf{u}^{\prime}=\left(u^{\prime}(x, y, z, t), v^{\prime}(x, y, z, t), w^{\prime}(x, y, z, t)\right)$ are the velocity components and $\mathrm{P}^{\prime}$ is the pressure in the perturbation field; $\overline{\mathbf{U}}$ is the flow field vectors from the baseflow. Following the work of Sheard et al. [11, 13], the power method was employed to extract the fastest growing instability mode at a specified wavelength $\lambda$. The wavelength of the instability along the axial direction is defined as $\lambda=2 \pi / k$, where $k$ is the axial wavenumber and varied between one and six in this investigation. The instability growth rate of the global flowfield $\sigma$ is nondimensionalised with the period of self-rotation, $\mathrm{T}=4 \pi^{2} \mathrm{~b}^{2} /\left(\Gamma^{+}+\Gamma^{-}\right)$. 

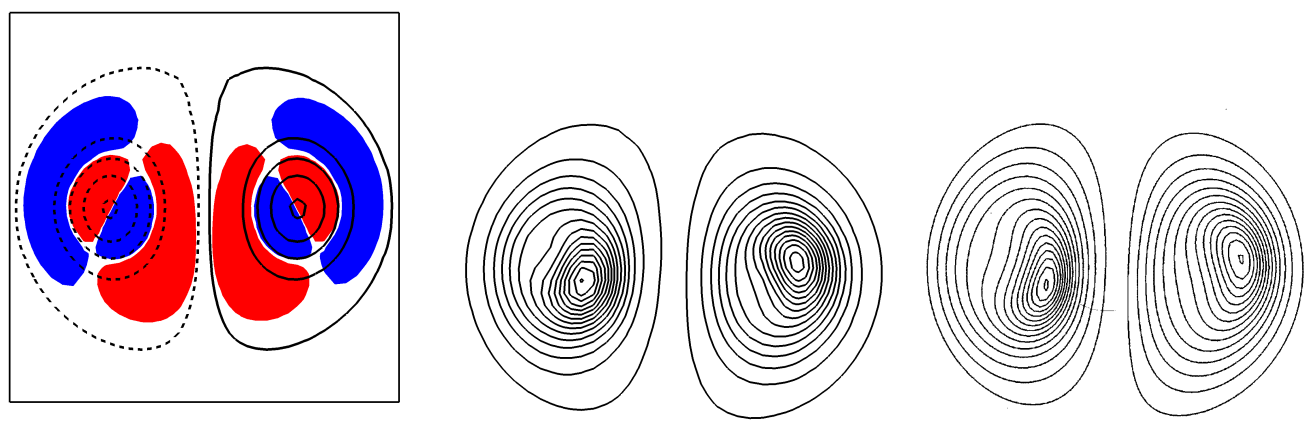

Figure 3: Left: Elliptic instability of a $\Lambda=-1$ vortex pair. Vorticity Perturbation field vorticity, $\omega_{p}$ (flooded contours) overlaid with baseflow vorticity (contour lines). Red and solid lines are positive vorticity, and blue and dashed lines represents negative. Normalised perturbation vorticity levels of $\omega_{p} / \omega_{0, \text { max }}^{+}= \pm 8.3 \times 10^{-5}$ are plotted; where $\omega_{0, \text { max }}^{+}$is the initial vorticity maxima of the positive vortex. Middle: Fourier reconstructed vorticity field of the left figure. Right: A corresponding three dimensional DNS result by Laporte [6]. Iso-contour lines are vorticity magnitudes. 


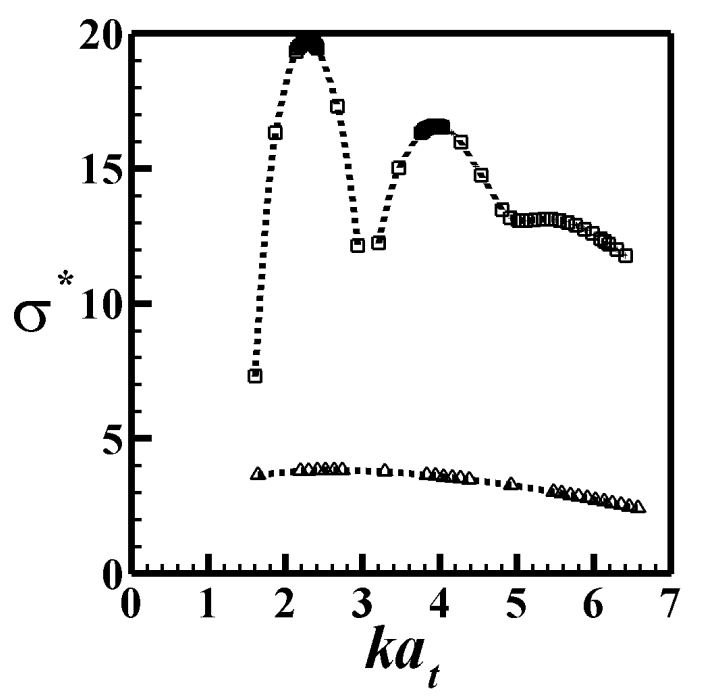

Figure 4: Computed non-dimensional growth rate comparison of the global instability for the different branches of vortex pairs $\Lambda=-0.19(\triangle)$ and $-0.685(\square)$. Dashed curves are added for guidance.

\section{$3 \quad$ Stability analysis results}

Two sets of the instability analysis results are considered in detail in this article, namely $\Lambda=-0.19$ and -0.685 . Both of these configurations are compared with the case of a CRES vortex pair. An example of the elliptic instabilities of a CRES vortex pair is shown in Figure 3, with a comparison between the current linear stability analysis prediction and the DNS result by Laporte \& Corjon [6]. The instability mode has previously been identifed as belonging to the first branch of the Kelvin mode coupling $(-1,1)$. The classification agrees with the experimental observations of Leweke \& Williamson [7] who described the instability modes as asymmetric and cooperative.

The non-dimensional growth rates, $\sigma^{*}=\sigma 4 \pi^{2} \mathrm{~b}^{2} /\left(\Gamma^{+}+\Gamma^{-}\right)$, of the global instability for $\Lambda=-0.19$ and -0.685 are plotted against the non-dimensional 

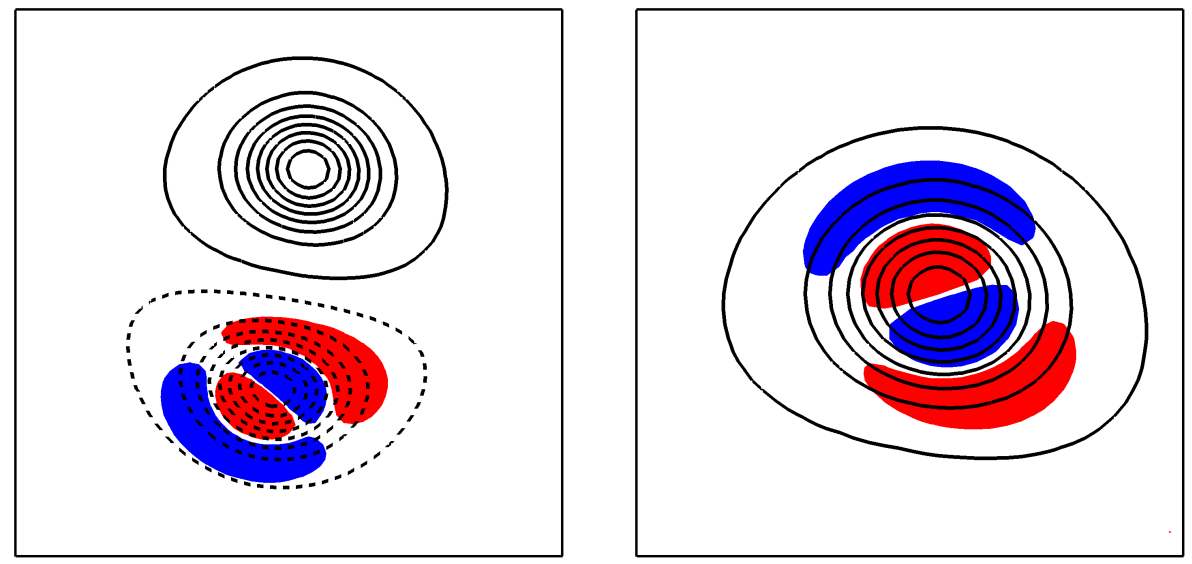

FiguRE 5: Left: Instability structure of a vortex pair with $\Lambda=-0.685$ for the first branch mode $(-1,1,1)$ on the negative vortex. Right: Re-scaled flooded contours for the positive vortex. Normalised perturbation vorticity levels of $\omega_{p} / \omega_{0, \max }^{+}= \pm 3 \times 10^{-4}$ are plotted for the left figure and $\pm 1.6 \times 10^{-5}$ for the right figure.

axial wavenumber $\mathrm{ka}$ in Figure 4 . For $\Lambda=-0.685$, three local peaks are clearly distinguishable; however, only a smooth curve lies across the entire range of $k a$ for $\Lambda=-0.19$, and overall the case of $\Lambda=-0.685$ have stronger global instability growth rates.

The instability structure of the first two branch modes for $\Lambda=-0.685$ are shown in Figures 5 and 6, which correspond to the non-dimensional axial wavenumbers, $\mathrm{ka}=2.3$ and 3.9, where the local peaks in the instability growth rate were identified, as shown in Figure 4. The vorticity perturbations were reflectively symmetric which caused the baseflow to yield asymmetric alterations, which were similar to the core deformations shown in Figure 3 (middle) for the CRES vortex pair.

The instability radial structure within the core became smaller in scale 

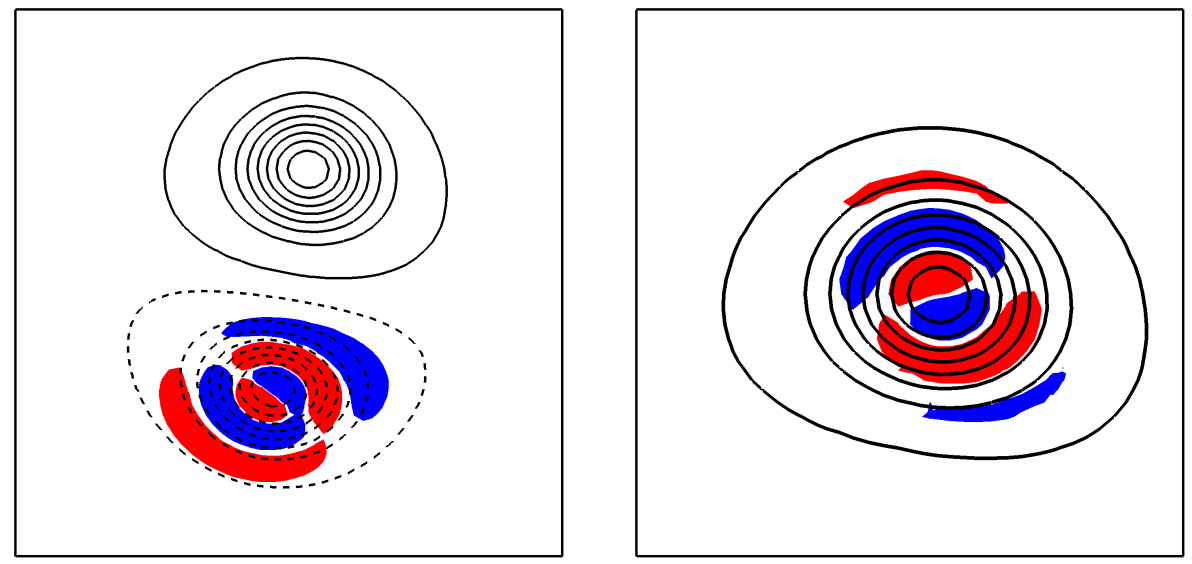

Figure 6: As per Figure 5 for the second branch mode $(-1,1,2)$. Normalised perturbation vorticity levels of $\omega_{\mathrm{p}} / \omega_{0, \max }^{+}= \pm 5 \times 10^{-4}$ are plotted for the left figure and $\pm 3.2 \times 10^{-6}$ for the right figure.

with the increment of the instability wavenumber $k$ (the decrement of wavelength $\lambda$ ) when comparing between the branches shown in Figures 5 and 6. The instability structures grew unevenly on the vortices of the unequalstrength vortex pairs. The amplitude of the instability was larger on the negative vortex than the positive vortex, which was due to the stronger induced strain from its counterpart with stronger circulation. The vorticity perturbation contours were re-scaled to a lower level on the positive vortex to show the structure of the perturbation within the vortex core.

For the vortex pair of $\Lambda=-0.19$, the instability structure was significantly different from the previous case. As with the case $\Lambda=-0.685$, Figure 7 shows two different modes corresponding to $k a=2.3$ and 4.2. According to the growth rate in Figure 4, no distinguishable branch was observed over a range of $k a$. The first mode $(k a=2.3)$ showed the property of reflective symmetry in the instability structure. But the instability structure in the negative vortex was extremely stretched around the positive vortex 

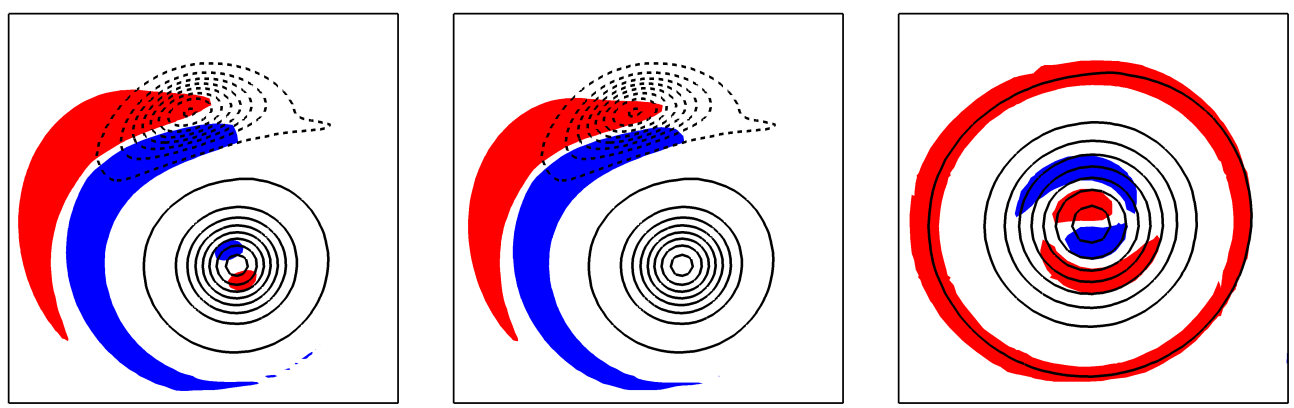

Figure 7: Left: The $k a=2.3$ mode of a vortex pair with $\Lambda=-0.19$. Middle: The $\mathrm{ka}=4.2$ mode on the negative vortex. Right: Re-scaled flooded contours for the positive vortex. Normalised perturbation vorticity levels of $\omega_{\mathrm{p}} / \omega_{0, \max }^{+}= \pm 5 \times 10^{-5}$ are plotted for the left figure, $\pm 8.3 \times 10^{-5}$ for the middle figure. and $\pm 3 \times 10^{-6}$ for the right figure.

and no longer concentrated in the vortex core. When comparing the first mode to the second mode for $\Lambda=-0.19$, the instability structure of the negative vortices had no apparent difference when ka was increased. But a smaller scale instability structure on the positive vortex was found to grow with increasing $\mathrm{ka}$. Based on the observed instability structure within the vortex cores, the second branch is believed to be a reflectively asymmetric mode for the $\Lambda=-0.19$ vortex pair.

\section{Conclusion}

The elliptic instabilities of a counter rotating vortex pair of unequal strength were shown to be different from what was known for an equal strength vortex pair. The highly deformed negative vortex in this study has been shown to alter the development of the instability. On the other hand, the instability mode shape observed in the positive vortex followed what was known previ- 
ously. Higher order radial modes were observed as ka was increased. The maximum predicted growth rate of the global instability mode for the vortex pair with $\Lambda=-0.685$ was approximately six times higher than for the pair with $\Lambda=-0.19$.

Acknowledgements The Dept. of Mechanical and Aerospace Engineering and the Fluids Laboratory for Aeronautical and Industrial Research of Monash University provided resources for this investigation; and the Australian Partnership for Advanced Computing enabled the investigation to take place.

\section{References}

[1] Batchelor, G. K., An Introduction to Fluid Dynamics, Cambridge University Press, 1967. C141

[2] Crow, S. C., Stability theory for a pair of trailing vortices, AIAA, 8(12), 2172-2179, 1970. C138

[3] Kelvin, L., Vibratons of a columnar vortex, Phil. Mag., 10, 155-168, 1880. C139

[4] Kerswell, R. R., Elliptical instability, Annu. Rev. Fluid Mech., 34, 83-113, 2002. doi:10.1146/annurev.fluid.34081701.171829 C138

[5] Lacaze, L., Ryan. K. \& Le Dizés, S., Elliptic instability in a strained Batchelor vortex, J. Fluid Mech., 577, 345-361, 2007. doi:10.1017/S0022112007004879 C141, C143

[6] Laporte, F. \& Corjon, A. Direct numerical simulations of the elliptic instability of a vortex pair, Phys. Fluids, 12(5), 1016-1031, 2000. doi:10.1063/1.870357 C138, C141, C144, C145 
[7] Leweke, T. \& Williamson, C. H. K., Cooperative elliptic instability of a vortex pair, J. Fluid Mech., 360, 85-119, 1998. C138, C139, C145

[8] Le Dizés, S. \& Verga, A., Viscous interactions of two co-rotating vortices before merging, J. Fluid Mech., 467, 389-410, 2002. doi:10.1017/S0022112002001532 C142

[9] Meunier, P. \& Leweke, T., Elliptic instability of a co-rotating vortex pair, J. Fluid Mech., 533, 125-159, 2005.

doi:10.1017/S0022112005004325 C138, C139

[10] Moore, D. W. \& Saffman, P. G., The instability of a straight vortex filament in a strain field, Proc. R. Soc. Lond. A., 346, 413-425, 1975. C138

[11] Sheard, G. J., Thompson, M. C. \& Hourigan, K., From spheres to circular cylinders: The stability and flow structures of bluff ring wakes, J. Fluid Mech., 492, 147-180, 2003. doi:10.1017/S002211200300512 $\mathrm{C} 143$

[12] Sheard, G. J., Leweke, T., Thompson, M. C. \& Hourigan, K., Flow around an impulsively arrested circular cylinder, Phys. Fluids, 19(8), 2007, 083601. doi:10.1063/1.2754346 C140

[13] Sheard, G. J. \& Ryan, K., Pressure-driven flow past spheres moving in a circular tube, J. Fluid Mech., 592, 233-262, 2007. doi:10.1017/S0022112007008543 C143

[14] So, J., Ryan, K. \& Sheard, G. J., Interaction of an unequal-strength vortex pair, In Proceedings of the 16th Australasian Fluid Mechanics Conference, Crown Plaza, Gold Coast, Queensland, Australia, 3-7 Dec 2007, 1457-1462. C139, C140, C142

[15] Spalart, P. R., 1998, Airplane trailing vortices, Annu. Rev. Fluid Mech., 30, 107-138. C138 
[16] Widnall, S. E. \& Sullivan, J. P., On the stability of vortex rings, Proc. Roy. Soc. A, 332, 335-353, 1973. C138

[17] Widnall, S. E., Bliss, D. B. \& Tasi, C.-Y., The instability of short waves on a vortex ring, J. Fluid Mech., 66, part 1, 35-47, 1974. C138

\section{Author addresses}

1. J. So, Fluids Laboratory for Aeronautical and Industrial Research, Department of Mechanical Engineering, Monash University, Victoria 3800, Australia.

2. K. Ryan, Fluids Laboratory for Aeronautical and Industrial Research, Department of Mechanical Engineering, Monash University, Victoria 3800, Australia.

3. G. J. Sheard, Fluids Laboratory for Aeronautical and Industrial Research, Department of Mechanical Engineering, Monash University, Victoria 3800, Australia; and Monash University Biomedical Engineering Alliance, Monash University, Victoria 3800, Australia 